\title{
Research on Polarization Cancellation of Nonstationary Ionosphere Clutter in HF Radar System
}

\author{
Xingpeng Mao, Hong Hong, Weibo Deng, and Yongtan Liu \\ School of Electronic and Information Engineering, Harbin Institute of Technology, Harbin, Heilongjiang 150001, China \\ Correspondence should be addressed to Xingpeng Mao; mxp@hit.edu.cn
}

Received 25 April 2014; Revised 20 August 2014; Accepted 9 September 2014

Academic Editor: Hang Hu

Copyright (C) 2015 Xingpeng Mao et al. This is an open access article distributed under the Creative Commons Attribution License, which permits unrestricted use, distribution, and reproduction in any medium, provided the original work is properly cited.

\begin{abstract}
Oblique projection polarization filter (OPPF) can be applied as an effective approach for interference cancellation in high-frequency surface wave radar (HFSWR) and other systems. In order to suppress the nonstationary ionosphere clutter further, a novel OPPF based clutter suppressing scheme is proposed in this paper. The polarization and nonstationary characteristic of the clutter are taken into account in the algorithms referred to as range-Doppler domain polarization suppression (RDDPS) and the range-time domain polarization suppression (RTDPS) method, respectively. The RDDPS is designed for weak ionosphere clutter and implemented in the range-Doppler domain directly, whereas the RTDPS algorithm is designed to suppress the powerful ionosphere clutter with a multisegment estimation and suppression scheme. About 15-23 dB signal to interference ratio (SIR) improvement can be excepted when using the proposed method, whereas the targets can be more easily detected in the range-Doppler map. Experimental results demonstrate that the scheme proposed is effective for nonstationary ionosphere clutter and is proven to be a practical interference cancellation technique for HFSWR.
\end{abstract}

\section{Introduction}

By exploiting the long-range propagation of vertically polarized electromagnetic wave in the band of $2-15 \mathrm{MHz}$, highfrequency surface wave radar (HFSWR) is able to receive vessel and low-flying aircraft echoes over the horizon. However, the signal environment of high-frequency (HF) band is far from satisfactory. The powerful shortwave radio interference, ionosphere clutter, and industrial interference that dominate the pure receiver noise in the HF band cause a significant limit of detection capability. Ionosphere clutter, which is often observed to mask multiple successive range and Doppler cells, is one of the main interference sources. In some cases, the power of the clutter is so high that even the target echoes are overwhelmed, resulting in poor detection and tracking performance $[1,2]$.

Adaptive beamforming schemes have been developed by using the space information of ionosphere clutter [35]. However, when the target is overlapped by ionosphere clutter from the same or close direction, such approaches are not ideal. According to the fact that the echoes of targets arriving along the surface of ocean used to be vertically polarized while ionosphere clutter is elliptically polarized, polarization filtering can be applied to improve the HF radar performance. By utilizing the polarization difference between the target and the clutter, polarization filter can be used to suppress interference [6-9]. However, a severe loss of coherent integration occurs when a conventional polarization filter is applied to coherent systems such as HFSWR, as an additional amplitude and/or phase distortion of the target signal will be introduced. In order to solve this problem, null phase-shift polarization filter (NPPF) [10] is proposed and then extended to a frequency domain null phase-shift multinotch polarization filter in $[11,12]$. Such improvement effectively keeps the temporal coherence of the target within coherent integration time (CIT) and successfully paved a way for the application of polarization filtering in HFSWR. To improve the flexibility and convenience, oblique projection polarization filter (OPPF) [13] is further proposed to deal with nonorthogonal signals. Successful experimental evaluation of OPPF for suppressing shortwave radio interference in HFSWR is reported in [14].

However, the ionosphere clutter cancellation method, which meets the requirement of practical HFSWR system, 
is still an open issue, as the ionosphere clutter is usually nonstationary. In this paper, two types of clutter cancellation approaches are given to suppress the nonstationary ionosphere clutter. The range-Doppler domain polarization suppression (RDDPS) is designed for weak ionosphere clutter and performed in the range-Doppler domain, while the range-time domain polarization suppression (RTDPS) is designed for strong ionosphere clutter. The procedure of clutter polarization estimation and clutter suppression in segments for the RTDPS is emphasized in our research. And the segmentation parameter optimization is also discussed in detail to obtain a balance between the clutter cancellation and the target restoration. The specific utilization of RDDPS or RTDPS depends on the type of the clutter at the corresponding range cell.

The remainder of this paper is organized as follows. In Section 2, the design of OPPF is introduced and the impact on the performance of the OPPF by the estimation error is analyzed. The nonstationary ionosphere clutter cancellation scheme is proposed in Section 3, whereas the applications of RDDPS and RTDPS are discussed in detail in Section 4. Then the experimental performance evaluation of the proposed scheme is given in Section 4. Finally, the conclusions are drawn in Section 5.

\section{Oblique Projection Polarization Filter}

2.1. Received Signal Model. Suppose the received wavefront is a completely polarized wave and its polarization angle and polarization angle difference are $\gamma$ and $\eta$, respectively. Ignoring the absolute phase, the incident signal $\mathbf{E}(t)$ can be described by Jones vector as in [10] as

$$
\begin{aligned}
\mathbf{E}(t) & =\left[\begin{array}{c}
S(t) \cos \gamma \\
S(t) \sin \gamma \exp (j \eta)
\end{array}\right] \\
& =\left[\begin{array}{c}
\cos \gamma \\
\sin \gamma \exp (j \eta)
\end{array}\right] S(t)=\mathbf{a} S(t),
\end{aligned}
$$

where $S(t) \cos \gamma$ is the horizontally polarized component of $\mathbf{E}(t)$ while $S(t) \sin \gamma \exp (j \eta)$ is the vertically polarized component of $\mathbf{E}(t)$. Vector $\mathbf{a}$ is defined as the polarization steering vector of $\mathbf{E}(t)$, and

$$
\mathbf{a}=\left[\begin{array}{c}
\cos \gamma \\
\sin \gamma e^{j \eta}
\end{array}\right]
$$

2.2. $O P P F$ and Error Analysis. Suppose the polarization steering vectors of the target and interference are $\mathbf{a}_{S}$ and $\mathbf{a}_{I}$, respectively. If the two steering vectors satisfy $\mathbf{a}_{S} \neq \mathbf{a}_{I}$, a corresponding polarization oblique projection operator can be constructed by [13]

$$
\mathbf{H}=\mathbf{a}_{S}\left(\mathbf{a}_{S}^{\mathrm{H}} \mathbf{P}_{\mathbf{a}_{I}}^{\perp} \mathbf{a}_{S}\right)^{-1} \mathbf{a}_{S}^{\mathrm{H}} \mathbf{P}_{\mathbf{a}_{I}}^{\perp},
$$

where $\mathbf{P}_{\mathbf{a}_{I}}^{\perp}=\mathbf{E}-\mathbf{a}_{I}\left(\mathbf{a}_{I}^{\mathrm{H}} \mathbf{a}_{I}\right)^{-1} \mathbf{a}_{I}^{\mathrm{H}}$ and $\mathbf{E}$ represents the unit matrix.

The fundamental property of this polarization oblique projection operator can be given as

$$
\mathrm{Ha}_{S}=\mathbf{a}_{S}, \quad \mathbf{H a}_{I}=0 .
$$

The scalar form of (4) can be given as

$$
\left(\mathbf{A}_{S}^{\dagger} \mathbf{H}\right) \mathbf{a}_{S}=1, \quad\left(\mathbf{A}_{S}^{\dagger} \mathbf{H}\right) \mathbf{a}_{I}=0,
$$

where $\mathbf{A}_{S}^{\dagger}=\left(\mathbf{a}_{S}^{\mathrm{H}} \mathbf{a}_{S}\right)^{-1} \mathbf{a}_{S}^{\mathrm{H}}$.

Usually, the received signal is a linear combination of the target $\mathbf{E}_{S}(t)=\mathbf{a}_{S} S(t)$, interference $\mathbf{E}_{I}(t)=\mathbf{a}_{I} I(t)$, and noise $\mathbf{n}(t)$. According to (4), the output of the OPPF can be given as

$$
\begin{aligned}
\widehat{\mathbf{E}}_{S}(t) & =\mathbf{H}\left(\mathbf{E}_{S}(t)+\mathbf{E}_{I}(t)+\mathbf{n}(t)\right) \\
& =\mathbf{H a}_{S} S(t)+\mathbf{H a}_{I} I(t)+\mathbf{H n}(t) \\
& =\mathbf{E}_{S}(t)+\mathbf{H n}(t) .
\end{aligned}
$$

When scalar form is required, the filtering process can be rewritten as

$$
\begin{aligned}
\widehat{S}(t) & =\mathbf{A}_{S}^{\dagger} \mathbf{H}\left(\mathbf{E}_{S}(t)+\mathbf{E}_{I}(t)+\mathbf{n}(t)\right) \\
& =\mathbf{A}_{S}^{\dagger} \mathbf{H} \mathbf{a}_{S} S(t)+\mathbf{A}_{S}^{\dagger} \mathbf{H} \mathbf{a}_{I} I(t)+\mathbf{A}_{S}^{\dagger} \mathbf{H n}(t) .
\end{aligned}
$$

According to (5), (7) can be simplified as

$$
\widehat{S}(t)=S(t)+\mathbf{A}_{S}^{\dagger} \mathbf{H n}(t) .
$$

Equations (6) and (8) indicate that the interference can be completely cancelled and the target is restored without distortion when the polarization steering vectors of the target and interference, which are utilized to build the subspaces of desired ranges and null spaces, are estimated accurately.

If the estimation accuracy is uncertain, in order to analyze the performance loss of OPPF that was caused by the estimation error, the mean square error (MSE) of the target can be defined as MSE $=\mathbf{E}\left\{\|\widehat{S}(t)-S(t)\|_{\mathrm{F}}^{2}\right\}$, where $\widehat{S}(t)$ is the recovered signal of the target $S(t)$ and $\mathbf{E}(\cdot)$ and $\|\cdot\|_{\mathrm{F}}$ denote the mathematical expectation and the Frobenius norm. Substituting (7) into MSE, the general form of MSE of the target $S(t)$ can be given as

$$
\mathrm{MSE}=\mathbf{E}\left\{\left\|\widehat{\mathbf{A}}_{S}^{\dagger} \widehat{\mathbf{H}} \mathbf{a}_{S} S(t)+\widehat{\mathbf{A}}_{S}^{\dagger} \widehat{\mathbf{H}} \mathbf{a}_{I} I(t)+\widehat{\mathbf{A}}_{S}^{\dagger} \widehat{\mathbf{H}} \mathbf{n}(t)-S(t)\right\|_{\mathbf{F}}^{2}\right\},
$$

where $\widehat{\mathbf{H}}=\widehat{\mathbf{a}}_{S}\left(\widehat{\mathbf{a}}_{S}^{\mathrm{H}} \mathbf{P}_{\widehat{\mathbf{a}}_{I}}^{\perp} \widehat{\mathbf{a}}_{S}\right)^{-1} \widehat{\mathbf{a}}_{S}^{\mathrm{H}} \mathbf{P}_{\widehat{\mathbf{a}}_{I}}^{\perp}, \widehat{\mathbf{A}}_{S}^{\dagger}=\left(\widehat{\mathbf{a}}_{S}^{\mathrm{H}} \widehat{\mathbf{a}}_{S}\right)^{-1} \widehat{\mathbf{a}}_{S}^{\mathrm{H}}, \mathbf{a}_{S}$ and $\mathbf{a}_{I}$ are the real polarization steering vectors of the target and the interference, and $\widehat{\mathbf{a}}_{S}$ and $\widehat{\mathbf{a}}_{I}$ are the estimated values, respectively.

Supposing the target signal, interference, and noise are uncorrelated, (9) can be simplified as

$$
\text { MSE }=|x|^{2} \sigma_{s}^{2}+|y|^{2} \sigma_{i}^{2}+|z|^{2} \sigma^{2},
$$

where

$$
\begin{gathered}
x=\widehat{\mathbf{A}}_{S}^{\dagger} \widehat{\mathbf{H}} \mathbf{a}_{S}-1, \\
y=\widehat{\mathbf{A}}_{S}^{\dagger} \widehat{\mathbf{H}}_{I}, \\
z=\sqrt{\frac{1}{\sin ^{2} \psi}}, \\
\psi=\arccos \left(\left|\widehat{\mathbf{a}}_{S}^{\mathrm{H}} \widehat{\mathbf{a}}_{I}\right|\right) .
\end{gathered}
$$


And $\sigma_{S}^{2}, \sigma_{I}^{2}$, and $\sigma^{2}$ are the power of the target, interference, and noise, respectively. Define $x$ and $y$ as the coefficients of $\sigma_{S}^{2}$ and $\sigma_{I}^{2}$. The error analysis of the MSE will be discussed in three cases as follows.

2.2.1. MSE without Estimation Error: $\widehat{\mathbf{a}}_{S}=\mathbf{a}_{S}$, $\widehat{\mathbf{a}}_{I}=\mathbf{a}_{I}$. Supposing the polarization steering vectors of the target and the interference are both accurately estimated, according to (11) and (12), we have $x=y=0$. The MSE gets to its minimum as

$$
\mathrm{MSE}=|z|^{2} \sigma^{2}
$$

2.2.2. MSE with Target Estimation Error: $\widehat{\mathbf{a}}_{S} \neq \mathbf{a}_{S}, \widehat{\mathbf{a}}_{I}=\mathbf{a}_{I}$. In this case, we have $x \neq 0$ and $y=0$, which indicates that the interference can be completely suppressed while the target is restored with distortion. Then the MSE can be simplified from (10) as

$$
\text { MSE }=|x|^{2} \sigma_{S}^{2}+|z|^{2} \sigma^{2} .
$$

Ignoring the noise term, the MSE is mainly determined by $x$ and $\sigma_{S}^{2}$. According to the properties of oblique projection [15], it is beneficial to decompose vector $\mathbf{a}_{S}$ into two specific components: the component that locates in the subspace spanned by $\widehat{\mathbf{a}}_{S}$ and the one which lies in the subspace spanned by $\mathbf{a}_{I}$. Therefore, $\mathbf{a}_{S}$ can be decomposed as

$$
\mathbf{a}_{S}=(\alpha+\beta) \widehat{\mathbf{a}}_{S}+\nu \mathbf{a}_{I},
$$

where

$$
\begin{gathered}
\alpha=\frac{\widehat{\mathbf{a}}_{S}^{\mathrm{H}} \mathbf{a}_{S}}{\left(\left|\widehat{\mathbf{a}}_{S}^{\mathrm{H}}\right|\left|\mathbf{a}_{S}\right|\right)}, \\
\beta=\frac{\left(\left|\mathbf{a}_{I}\right|^{2} \widehat{\mathbf{a}}_{S}^{\mathrm{H}} \mathbf{d}-\widehat{\mathbf{a}}_{S}^{\mathrm{H}} \mathbf{a}_{I} \mathbf{a}_{I}^{\mathrm{H}} \mathbf{d}\right)}{\left(\left|\widehat{\mathbf{a}}_{S}\right|^{2}\left|\mathbf{a}_{I}\right|^{2}-\widehat{\mathbf{a}}_{S}^{\mathrm{H}} \mathbf{a}_{I} \mathbf{a}_{I}^{\mathrm{H}} \widehat{\mathbf{a}}_{S}\right)}, \\
\nu=\frac{\left(\left|\widehat{\mathbf{a}}_{S}\right|^{2} \mathbf{a}_{I}^{\mathrm{H}} \mathbf{d}-\mathbf{a}_{I}^{\mathrm{H}} \widehat{\mathbf{a}}_{S} \widehat{\mathbf{a}}_{S}^{\mathrm{H}} \mathbf{d}\right)}{\left(\left|\widehat{\mathbf{a}}_{S}\right|^{2}\left|\mathbf{a}_{I}\right|^{2}-\widehat{\mathbf{a}}_{S}^{\mathrm{H}} \mathbf{a}_{I} \mathbf{a}_{I}^{\mathrm{H}} \widehat{\mathbf{a}}_{S}\right)}, \\
\mathbf{d}=\mathbf{a}_{S}-\alpha \widehat{\mathbf{a}}_{S} .
\end{gathered}
$$

According to [13], the first part in (17), which locates in the target subspace, will be reserved as a whole, whereas the second part will be cancelled by $\widehat{\mathbf{H}}$ to null. Substituting (17) into (11), $x$ can be rewritten as

$$
x=\widehat{\mathbf{A}}_{S}^{\dagger} \widehat{\mathbf{H}}\left((\alpha+\beta) \widehat{\mathbf{a}}_{S}+v \mathbf{a}_{I}\right)-1=\alpha+\beta-1 .
$$

Therefore, (16) can be rewritten as

$$
\text { MSE }=|\alpha+\beta-1|^{2} \sigma_{S}^{2}+|z|^{2} \sigma^{2} .
$$

Equation (20) indicates that the MSE is mainly affected by the term of $|\alpha+\beta-1|^{2} \sigma_{S}^{2}$, which is the product of the target power $\sigma_{S}^{2}$ and its corresponding coefficient $x$. The lower the estimation accuracy of target's polarization state is, the larger the value of $x$ will be, and the MSE increases. However, when the target is estimated with no error, (20) will be identical to (15).
2.2.3. MSE with Interference Estimation Error: $\widehat{\mathbf{a}}_{S}=\mathbf{a}_{S}, \widehat{\mathbf{a}}_{I} \neq$ $\mathbf{a}_{I}$. Supposing the target signal is accurately estimated while the interference is not, we have $\widehat{\mathbf{A}}_{S}^{\dagger} \widehat{\mathbf{H}} \mathbf{a}_{S}=1$ and $\widehat{\mathbf{A}_{S}^{\dagger}} \widehat{\mathbf{H}} \mathbf{a}_{I} \neq 0$, which makes $x=0, y \neq 0$. In this case, the target is perfectly restored while the interference is partly suppressed by OPPF. Decomposing vector $\mathbf{a}_{I}$ into two specific components, we have

$$
\mathbf{a}_{I}=\left(\alpha^{\prime}+\beta^{\prime}\right) \widehat{\mathbf{a}}_{I}+v^{\prime} \mathbf{a}_{S},
$$

where

$$
\begin{gathered}
\alpha^{\prime}=\frac{\widehat{\mathbf{a}}_{I}^{\mathrm{H}} \mathbf{a}_{I}}{\left(\left|\widehat{\mathbf{a}}_{I}^{\mathrm{H}}\right|\left|\mathbf{a}_{I}\right|\right)}, \\
\beta^{\prime}=\frac{\left(\left|\mathbf{a}_{S}\right|^{2} \widehat{\mathbf{a}}_{I}^{\mathrm{H}} \mathbf{d}-\widehat{\mathbf{a}}_{I}^{\mathrm{H}} \mathbf{a}_{S} \mathbf{a}_{S}^{\mathrm{H}} \mathbf{d}^{\prime}\right)}{\left(\left|\widehat{\mathbf{a}}_{I}\right|^{2}\left|\mathbf{a}_{S}\right|^{2}-\widehat{\mathbf{a}}_{I}^{\mathrm{H}} \mathbf{a}_{S} \mathbf{a}_{S}^{\mathrm{H}} \widehat{\mathbf{a}}_{I}\right)}, \\
\nu^{\prime}=\frac{\left(\left|\widehat{\mathbf{a}}_{I}\right|^{2} \mathbf{a}_{S}^{\mathrm{H}} \mathbf{d}-\mathbf{a}_{S}^{\mathrm{H}} \widehat{\mathbf{a}}_{I} \widehat{\mathbf{a}}_{I}^{\mathrm{H}} \mathbf{d}^{\prime}\right)}{\left(\left|\widehat{\mathbf{a}}_{I}\right|^{2}\left|\mathbf{a}_{S}\right|^{2}-\widehat{\mathbf{a}}_{I} \mathbf{a}_{S} \mathbf{a}_{S}^{\mathrm{H}} \widehat{\mathbf{a}}_{I}\right)}, \\
\mathbf{d}^{\prime}=\mathbf{a}_{I}-\boldsymbol{\alpha}^{\prime} \widehat{\mathbf{a}}_{I} .
\end{gathered}
$$

Substituting (21) into (12), $y$ can be rewritten as

$$
y=\widehat{\mathbf{A}}_{S}^{\dagger} \widehat{\mathbf{H}}\left(\alpha^{\prime}+\beta^{\prime}\right) \widehat{\mathbf{a}}_{I}+\widehat{\mathbf{A}}_{S}^{\dagger} \widehat{\mathbf{H}} \nu^{\prime} \mathbf{a}_{S}=v^{\prime} .
$$

The MSE can be simplified as

$$
\begin{aligned}
\mathrm{MSE} & =\left|\nu^{\prime}\right|^{2} \sigma_{I}^{2}+|z|^{2} \sigma^{2} \\
& =\sigma_{S}^{2}\left(\frac{\left|\nu^{\prime}\right|^{2}}{\mathrm{SIR}}+\frac{|z|^{2}}{\mathrm{SNR}}\right),
\end{aligned}
$$

where SIR $=\sigma_{S}^{2} / \sigma_{I}^{2}$ and $\mathrm{SNR}=\sigma_{S}^{2} / \sigma^{2}$ refers to the signal to noise ratio (SNR).

Equation (24) demonstrates that the interference cannot be suppressed completely when it is estimated with error. The degree of interference suppression is determined by the component $\left(\alpha^{\prime}+\beta^{\prime}\right) \widehat{\mathbf{a}}_{I}$ in (21). The smaller the estimation deviation is, the larger this component will be and the performance of OPPF will be better.

Moreover, fixing the SIR and SNR and the power of the target, according to (14), it can be noticed that the part of $|z|^{2} \sigma^{2}$ in (15), (20), and (24) will increase and the MSE will become larger, when the polarization state of the target and the interference are getting close.

\section{Polarization Cancellation for Ionosphere Clutter}

To apply an OPPF in ionosphere clutter cancellation, it is necessary to accurately estimate the polarization state of the target and the ionosphere clutter, so that the clutter can be suppressed to the maximum whereas the target can be effectively restored. With regard to the nonstationary characteristic of the ionosphere clutter, the polarization estimation methods and ionosphere clutter cancellation scheme should be properly designed. 
3.1. Polarization and Nonstationary Characteristic of Ionosphere Clutter. Ionosphere clutter, which is a kind of selfgenerated interference in HFSWR, is the radar signals reflecting back from the ionosphere. The polarization plane of the electromagnetic wave is usually rotated by the ionosphere along the radio propagation path, which makes the high power vertically polarized radar signals change into elliptically polarized ones. However, the echoes of targets arriving along the surface of ocean remain vertically polarized. Evident difference between the elliptical polarized ionosphere clutters and the vertically polarized target signals can be found in the dual-polarized HFSWR. That is, the power of the horizontally polarized component of ionosphere clutter is close to that of the vertically polarized part or even stronger. However, the power of the horizontally polarized component of the target echoes propagating along the ocean surface is quite weak compared with that of the vertically polarized component $[16,17]$.

Affected by the irregular activities of the free electrons and charged ions, the electron concentration of the ionosphere is unstable, which leads to the nonstationary feature of the ionosphere clutter [3]. The following characteristics of the ionosphere clutter are usually observed.

(1) The ionosphere clutter is not stable in a CIT, and its duration time varies from several pulse periods to the whole CIT.

(2) In each single pulse, some clutters occupy less range cells whereas some occupy more.

(3) The nonstationary feature also leads to the different frequency spread in Doppler spectrum; some clutters locate in multiple successive Doppler cells while some exist in fewer Doppler cells.

(4) The ionosphere clutter is usually elliptically polarized but its polarization state is unstable in the whole CIT [5]. As a result, it behaves as a partially polarized wave in a long time observation.

3.2. Nonstationary Ionosphere Clutter Polarization Cancellation Scheme. According to the characteristics of ionosphere clutter discussed above, a novel OPPF based polarization cancellation scheme for nonstationary ionosphere clutter is proposed. Figure 1 gives the basic structure of this scheme. In Figure 1, the raw data is pretreated first to obtain the polarization information of the clutters and the targets, which is necessary for building the RDDPS or RTDPS; then the signals are filtered in range or range-Doppler domain, and the output of the filter will be utilized to perform range and Doppler processing in RTDPS or given as the result in RDDPS, respectively. Five steps are needed to finish the clutter cancellation.

(1) Because of the weak power of radar echoes, range processing and Doppler processing need to be carried out first. Range-Doppler maps are generated from the raw data of each horizontal and vertical channel, respectively.

(2) The state of all the samples in the range-Doppler map is then estimated, including the power value and

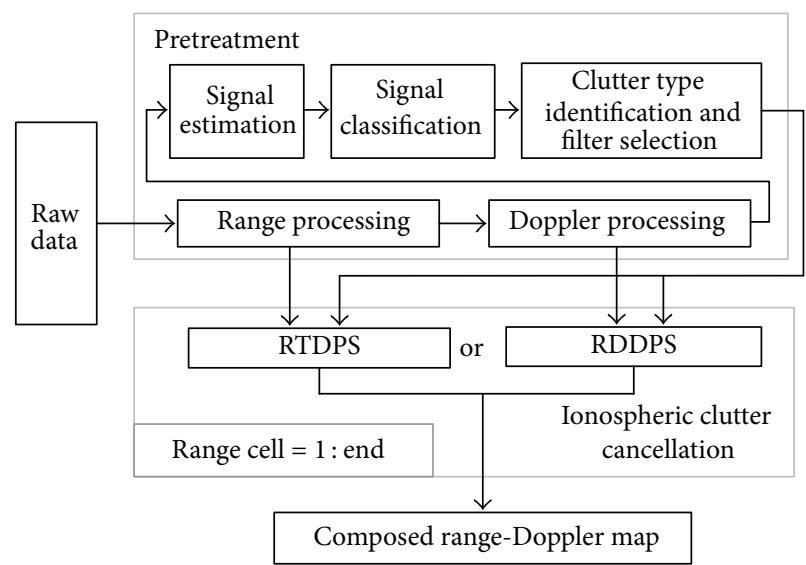

FIGURE 1: Structure of ionosphere clutter cancellation scheme.

polarization parameters. By using the basic characteristic of the signals in the range-Doppler map, the target and other clutters, such as ground clutter, ocean clutter, and ionosphere clutter, can be preliminarily separated and classified.

(3) Based on the power estimation, the type of ionosphere clutter is identified cell by cell in range domain. The specific utilization of RDDPS or RTDPS at each range cell is determined by the clutter type at each range cell.

(4) RDDPS and RTDPS algorithms, designed for different types of ionosphere clutter, will be used to suppress the clutter. The former one is designed for the ionosphere clutter with low power and the filtering process is carried out in range-Doppler domain directly. The latter one is designed for powerful clutter, and the filtering process is mainly carried out in the range-time domain. In a practical HFSWR system, the two types of ionosphere clutter may exist simultaneously and locate at different range cells. Therefore, the selection of RDDPS or RTDPS is decided by the clutter type at the corresponding range cell. Because of the adaptive scheme in this part, an optimal filtering effect can be obtained.

(5) After all the range cells are treated with the procedure in (4), the final range-Doppler map is composed for RDDPS, whereas a range-Doppler processing is needed for RTDPS to obtain the final range-Doppler map.

3.3. Pretreatment. As drawn in Figure 1, pretreatment includes the range-Doppler processing, the signal parameters estimation, signal classification, determination of ionosphere clutter type, and the selection of filtering method. Different from conventional processing, in a dual-polarized HFSRW system, the radar raw data of both the horizontal polarization channel and vertical polarization channel are recorded simultaneously. Then range-Doppler map of each channel can be obtained, respectively. Then the power value and polarization state of all the signals in the range-Doppler map can be estimated and stored. After all the signals are 
classified, the target (big enough or separated from the clutter), ground/ocean clutter, and ionosphere clutter will be separated apart. Suppose the range-Doppler map is composed by $J$ range cells and $R$ Doppler cells. The samples of the vertical and horizontal polarization channel at the current range-Doppler cell $(j, r)$ are defined as $V(j, r)$ and $H(j, r)$, where $1 \leq j \leq J$ and $1 \leq r \leq R$, respectively. The estimation values of the power value $d(j, r)$, polarization angle $\gamma(j, r)$, and polarization angle difference $\eta(j, r)$ of range-Doppler cell $(j, r)$ can be given as

$$
\begin{gathered}
\widehat{d}(j, r)=\sqrt{(V(j, r))^{2}+(H(j, r))^{2}}, \\
\widehat{\gamma}(j, r)=\arctan \left(\frac{\operatorname{abs}(V(j, r))}{\operatorname{abs}(H(j, r))}\right), \\
\widehat{\eta}(j, r)=\operatorname{angle}(V(j, r))-\operatorname{angle}(H(j, r)),
\end{gathered}
$$

where angle() is the function to obtain the complex phase of $V(j, r)$ and $H(j, r)$.

After the parameters have been estimated, all the signals will be classified. Firstly, a power value gate is set by $d_{L}$ to reduce the impact of noise. All the samples that satisfy $\widehat{d}(j, r) \geq d_{L}$ are determined to be valid while the other samples are considered to be noise. Secondly, the ocean clutter can be separated by utilizing the relationship between the radar transmitting frequency and the Braggs frequency [18]. Thirdly, by using the polarization characteristic of the ionosphere clutter, a polarization angle gate $\gamma_{L}$ is further defined. Those valid samples that satisfy $\widehat{\gamma}(j, r) \leq \gamma_{L}$ are determined as ionosphere clutter while the others are considered as echoes returning along the ocean and/or the mixed signal. In this way, the target, ocean clutter, and ionosphere clutter are preliminarily separated from each other in the range-Doppler map.

The ionosphere clutter is checked cell by cell. For the range cells that ionosphere clutter exists in, polarization cancellation scheme will be applied. In order to decide which polarization filtering algorithm will be taken, define function Fn, of which the logic is set to $\{1,0\}$. If Fn $=1$, RDDPS algorithm is chosen; if Fn $=0$, RTDPS algorithm will be applied.

The criterion of algorithm selection is based on the relationship of the power between the target and the ionosphere clutter. At the corresponding range cell, if the maximum value of the ionosphere clutter power is smaller than the average power of the target, the clutter is considered to be weak and Fn $=1$. On the contrary, the clutter is considered to be strong and $\mathrm{Fn}=0$. Suppose the set of power values of all the ionosphere clutters and targets at the corresponding range cell are defined as $D_{I}$ and $D_{S}$, respectively; Fn can be specified as

$$
\text { Fn }= \begin{cases}1, & \max \left(D_{I}\right)<\operatorname{mean}\left(D_{S}\right) \\ 0, & \max \left(D_{I}\right) \geq \operatorname{mean}\left(D_{S}\right)\end{cases}
$$

However, in certain range cells, the target may be nonexistent; the value of Fn in (26) will be uncertain. Therefore,

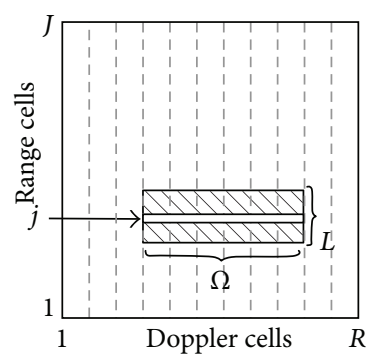

FIGURE 2: Diagrammatic illustration of RDDPS.

considering the Bragg scattering lines have the similar polarization characteristic with the target, (26) can be modified as

$$
\text { Fn }= \begin{cases}1, & \max \left(D_{I}\right)<\max \left(D_{\mathrm{Bragg}, L}, D_{\mathrm{Bragg}, R}\right) \\ 0, & \max \left(D_{I}\right) \geq \max \left(D_{\mathrm{Bragg}, L}, D_{\mathrm{Bragg}, R}\right)\end{cases}
$$

where $D_{\mathrm{Bragg}, L}$ and $D_{\mathrm{Bragg}, R}$ are the power value of left and right Braggs at the current range cell, respectively.

3.4. Range-Doppler Domain Polarization Suppression. To illustrate the potential advantages of range-Doppler domain suppression, three main aspects are given as follows.

(1) Due to the time-frequency invariance of the signal's polarization characteristic $[12,19]$, the estimation of the signal's polarization parameters can be performed in range-Doppler domain.

(2) In range-Doppler domain the power of each signal can be concentrated on limited range and Doppler cells, respectively, which significantly increases the SNR and interference to noise ratio (INR). In this way, the polarization estimation accuracy of both the target and the clutter will be improved effectively. As mentioned in Section 2, the improvement of estimation accuracy is beneficial for the clutter suppression and the restoration of the targets as well.

(3) In range-Doppler domain, the multiple clutters and targets can be isolated in different cells, providing a possibility of individual polarization estimation and suppression for each clutter, and the polarization degree of the signals is improved.

Based on the analysis above, polarization filtering in range-Doppler domain is considered to be more suitable to process the ionosphere clutter with weak power. The improved SIR and INR will significantly enhance the estimation accuracy and the performance of the filter.

The illustration of RDDPS is drawn in Figure 2. Supposing there are some ionosphere clutters existing at the range cell $j$, a range-Doppler window $(L \times \Omega)$ is first set in the rangeDoppler map $(J \times R)$, as shown in Figure 2. In the center of the window is the clutter area to be processed. The polarization state of clutters at the range cell $j$ will be estimated in the adjacent cells, as the characteristic of the ionosphere clutter in the neighbor range-Doppler is similar [3]. 
In the range-Doppler window, the polarization estimation is performed cell by cell in range for $L$ times. In each range cell, the samples that have the maximum power value are used for polarization estimation. To avoid the influence of target-like signal, the range-Doppler domain data in the window are excluded with the target and ground/ocean clutter. In this way, a sum of $L$ polarization parameters is obtained and the corresponding $L$ OPPF operators are generated. Ignoring the noise and supposing there are $Q$ clutters at range cell $j$, the composite input signal $\mathbf{E}(j, \omega)$ can be expressed as

$$
\begin{aligned}
\mathbf{E}(j, \omega) & =\mathbf{E}_{S}(j, \omega)+\mathbf{E}_{I}(j, \omega) \\
& =\mathbf{E}_{S}(j, \omega)+\sum_{q=1}^{Q} \mathbf{E}_{I, q}(j, \omega) \\
& =\mathbf{a}_{S} S(j, \omega)+\sum_{q=1}^{Q} \mathbf{a}_{I, q} I_{q}(j, \omega),
\end{aligned}
$$

where $\omega$ is the Doppler frequency, $\mathbf{E}_{S}(j, \omega)$ and $\mathbf{E}_{I}(j, \omega)$ are the target and the ionosphere clutters at range cell $j$, and $\mathbf{E}_{I, q}(j, \omega)$ is the $q$ th clutter of $\mathbf{E}_{I}(j, \omega)$, where $\mathbf{a}_{S}, \mathbf{a}_{I, q}, S(j, \omega)$, and $I_{q}(j, \omega)$ are the polarization steering vector and rangeDoppler spectrum of the target and the qth clutter $\mathbf{E}_{I, q}(j, \omega)$, respectively.

For simplicity, suppose $L=Q$ and the generated $L$ OPPF operators have one-to-one correspondence with the $Q$ clutters. Specifically, the $l$ th OPPF operator $\mathbf{H}_{l}$ is generated to suppress the qth clutter, when $l=q$. According to (3), the corresponding OPPF operator can be given as $\mathbf{H}_{l}=$ $\mathbf{a}_{S}\left(\mathbf{a}_{S}^{\mathrm{H}} \mathbf{P}_{\mathbf{a}_{I, l}}^{\perp} \mathbf{a}_{S}\right)^{-1} \mathbf{a}_{S}^{\mathrm{H}} \mathbf{P}_{\mathbf{a}_{I, l}}^{\perp}, l=1,2, \ldots, L$. According to (6), the output of $l$ th OPPF at range cell $j$ can be expressed as

$$
\begin{aligned}
\mathbf{Y}_{l}(j, \omega)= & \mathbf{H}_{l} \mathbf{E}(j, \omega) \\
= & \mathbf{H}_{l}\left(\mathbf{a}_{S} S(j, \omega)+\mathbf{a}_{I, l} I_{l}(j, \omega)\right. \\
& \left.+\sum_{q=1}^{l-1} \mathbf{a}_{I, q} I_{q}(j, \omega)+\sum_{q=l+1}^{Q} \mathbf{a}_{I, q} I_{q}(j, \omega)\right) \\
= & \mathbf{E}_{S}(j, \omega)+\mathbf{H}_{l} \sum_{q=1, q \neq l}^{Q} \mathbf{E}_{I, q}(j, \omega),
\end{aligned}
$$

where $\mathbf{Y}_{l}(j, \omega)$ is the $l$ th range-Doppler spectrum, in which the $l$ th ionosphere clutter $\mathbf{E}_{I, l}(j, \omega)$ is completely suppressed.

Equation (29) indicates that the $l$ th clutter $\mathbf{E}_{I, l}(j, \omega)$ can be effectively cancelled by $\mathbf{H}_{l}$ while the rest of $Q-1$ clutters are still left in $\mathbf{Y}_{l}(j, \omega)$. Obviously, when all OPPF operators are applied to $\mathbf{E}_{I}(j, \omega)$ in turn, $L$ filtering results will be obtained and only the clutter that matches $\mathbf{a}_{I, l}$ will be suppressed to the maximum. Suppose all the clutters are not overlapping with each other in the Doppler spectrum. In order to obtain a composite result from the above $L$ filtering results, logic product process [12], which reserves the smallest points in
Doppler spectrum from the $L$ filtering results, can be applied. Therefore, all the $Q$ ionosphere clutters will be suppressed simultaneously in the Doppler spectrum. Moreover, as shown in (29), the target $\mathbf{E}_{S}(j, \omega)$ can be completely restored in each filtering result. In this way, the logic product process will not influence $\mathbf{E}_{S}(j, \omega)$ in the final result. And the output of $\operatorname{RDDPS} \mathbf{Y}_{\mathrm{RDDPS}}(j, \omega)$ can be expressed as

$$
\mathbf{Y}_{\mathrm{RDDPS}}(j, \omega)=\sum_{r=1}^{R}\left\{\delta\left(\omega-\omega_{r}\right) \min _{l=1}^{L}\left(\mathbf{H}_{l} \mathbf{E}(j, \omega)\right)\right\},
$$

where $\omega_{r}$ is the Doppler frequency of Doppler cell $r$.

When the actual number of the clutters is less than $L$, ( $L>Q$ ), which is the most common situation for RDDPS, the amount of estimated parameters is more than the amount of clutters. Clearly, for some clutters, their polarization state will be estimated for more than once in the rangeDoppler window. And the corresponding filtering results will be similar. Note that the OPPF, which is generated by the estimated parameters approaching the real values, will suppress the clutter deeper; the recovered $\mathbf{Y}_{\mathrm{RDDPS}}(j, \omega)$ in fact can be considered as an optimized result of the $L$ filter outputs.

3.5. Range-Time Domain Polarization Suppression. The scheme of RDDPS takes advantage of the characteristic of the ionosphere clutter in range-Doppler domain and hence is suitable for ionosphere clutter cancellation of low power in HFSWR. However, the nonstationary characteristic of ionosphere clutter will degrade the performance of RDDPS, as a result of the decline of clutter's polarization degree. To solve this problem, another scheme which is referred to as range-time domain polarization suppression (RTDPS) is proposed.

Rather than estimating the polarization state in a CIT, the nonstationary characteristic of ionosphere clutter can be relaxed when the clutter is observed under a smaller time scale. By dividing the time domain data into many segments, the polarization degree in each segment can be improved. Thus, for a RTDPS, data segmentation is performed prior to the estimation and filtering process. The range-Doppler map is used to find the position (range and Doppler frequency) of the clutter, and the polarization state of the clutter is estimated in the corresponding range-Doppler cell of each segment, whereas the filtering process is performed in the range-time domain. To guarantee the estimation accuracy, a higher INR is required to compensate the decreased coherent integration time in each segment. That is why the RTDPS is more suitable for suppressing the strong ionosphere clutters. For the case of single clutter in the corresponding range cell, RTDPS is applied in the mode of single-notch mode, whereas a multinotch filtering mode of RTDPS will be taken when more clutters exist. It is necessary to emphasize that the notch is in the Doppler domain rather than in the polarization domain, which is completely different from the definition in [12].

3.5.1. Single-Notch RTDPS. As shown in Figure 3, the singlenotch RTDPS contains the following parts: setting of notch 
in Doppler domain, time domain segmentation, polarization estimation and clutter suppressing in each segment, and the final time domain data reconstruction.

Based on the results of the pretreatment step, a Doppler notch is set first to cover the region of several successive Doppler cells where the ionosphere clutter locates. In this way, the polarization steering vector of the ionosphere clutter can be estimated from the samples in the Doppler notch. After being processed by RTDPS, the ionosphere clutter in this area will be suppressed heavily, forming a "notch" in the Doppler spectrum.

After the Doppler notch has been set, the time domain data is divided into $P$ segments. In each segment, Doppler processing and polarization estimation are carried out to generate the corresponding OPPF operator. As shown in Figure 3, the ionosphere clutters in the Doppler notch will be suppressed by the OPPF within each segment. It should be noticed that the polarization estimation is finished in Doppler domain, whereas the filter is used in time domain.

Suppose the sum of time domain samples at the current range cell $j$ is $N$ and the number of segments is $P$ (suppose $N$ can be exactly divided by $P$ ). The ionosphere clutter $\mathbf{E}_{I}(j, n)$ can be described as

$$
\mathbf{E}_{I}(j, n)=\sum_{p=1}^{P} \mathbf{E}_{I, p}(j, n), \quad \frac{N(p-1)}{P}<n \leq \frac{N p}{P},
$$

where $\mathbf{E}_{I, p}(j, n)$ is the clutter in segment $p$ in range-time domain

In each segment, it is convenient to decompose $\mathbf{E}_{I, p}(j, n)$ into the completely polarized component $\mathbf{E}_{I_{-} C P, p}(j, n)$ and the completely unpolarized component $\mathbf{E}_{I_{-} U \mathrm{UP}, p}(j, n) ; \mathbf{E}_{I}(j, n)$ can be rewritten as

$$
\begin{array}{r}
\mathbf{E}_{I}(j, n)=\sum_{p=1}^{P}\left(\mathbf{E}_{I_{-} \mathrm{CP}, p}(j, n)+\mathbf{E}_{I_{-} \mathrm{UP}, p}(j, n)\right) \\
=\sum_{p=1}^{P}\left(\mathbf{a}_{I, p} I_{p}(j, n)+\mathbf{E}_{I_{-} \mathrm{UP}, p}(j, n)\right), \\
\frac{N(p-1)}{P}<n \leq \frac{N p}{P},
\end{array}
$$

where $\mathbf{a}_{I, p}$ and $I_{p}(j, n)$ are the polarization steering vector and the corresponding time domain data of the completely polarized component $\mathbf{E}_{I_{-} \mathrm{CP}, p}(j, n)$.

Meanwhile, the highly polarized target $\mathbf{E}_{S}(j, n)$ can be described as

$$
\begin{array}{r}
\mathbf{E}_{S}(j, n)=\sum_{p=1}^{P} \mathbf{E}_{S, p}(j, n)=\sum_{p=1}^{P} \mathbf{a}_{S} S_{p}(j, n), \\
\frac{N(p-1)}{P}<n \leq \frac{N p}{P},
\end{array}
$$

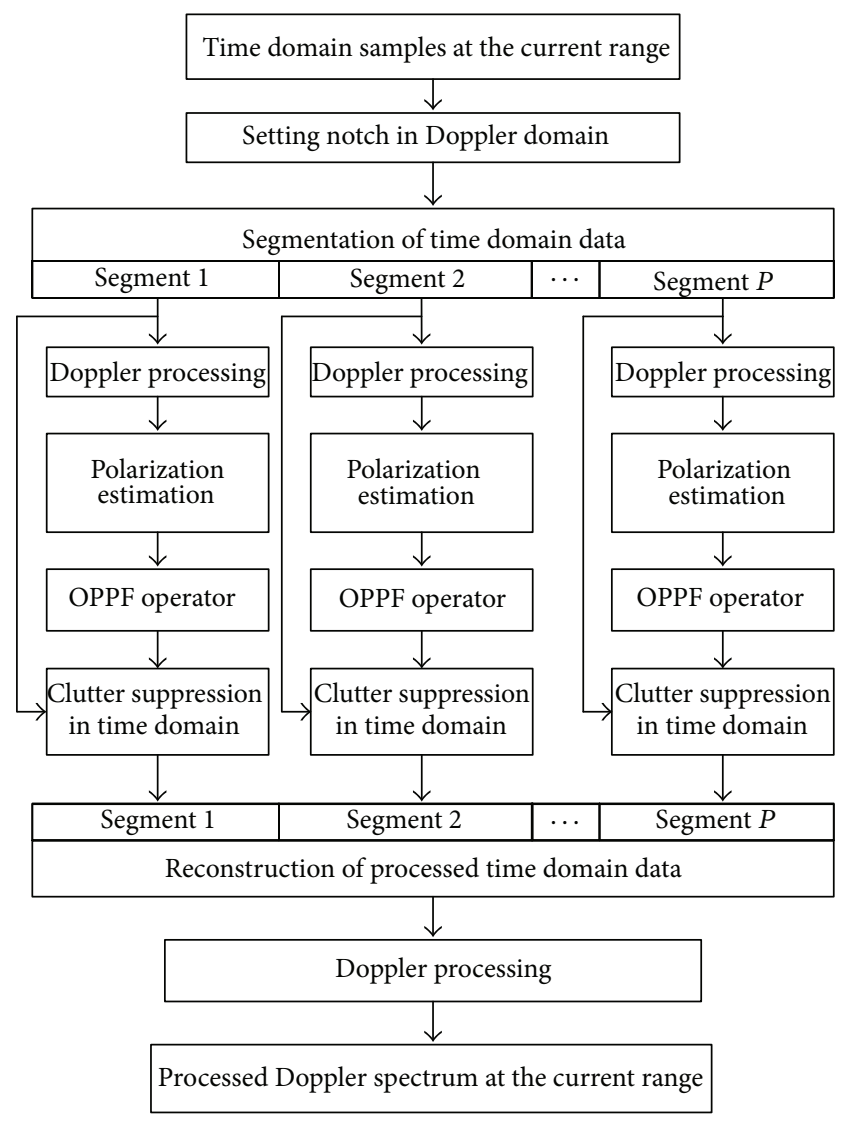

Figure 3: Principle of single-notch RTDPS.

where $\mathbf{a}_{S}$ is the polarization steering vector of the target and $S_{p}(j, n)$ is the corresponding time domain data of $\mathbf{E}_{S, p}(j, n)$ in segment $p$, respectively.

Then the OPPF operators of each segment can be obtained as $\mathbf{H}_{p}=\mathbf{a}_{S}\left(\mathbf{a}_{S}^{\mathrm{H}} \mathbf{P}_{\mathbf{a}_{I, p}}^{\perp} \mathbf{a}_{S}\right)^{-1} \mathbf{a}_{S}^{\mathrm{H}} \mathbf{P}_{\mathbf{a}_{I, p}}^{\perp}$, where $\mathbf{P}_{\mathbf{a}_{I, p}}^{\perp}=\mathbf{I}-$ $\mathbf{a}_{I, p}\left(\mathbf{a}_{I, p}^{\mathrm{H}} \mathbf{a}_{I, p}\right)^{-1} \mathbf{a}_{I, p}^{\mathrm{H}}$ and $p=1,2, \ldots, P$. According to (6), the filtering process of single-notch RTDPS can be given as

$$
\begin{array}{r}
\widehat{\mathbf{E}}_{S}(j, n)=\sum_{p=1}^{P}\left\{\mathbf{H}_{p}\left(\mathbf{E}_{S, p}(j, n)+\mathbf{E}_{I, p}(j, n)\right)\right\} \\
=\sum_{p=1}^{P}\left\{\mathbf{H}_{p} \mathbf{a}_{S} S_{p}(j, n)+\mathbf{H}_{p} \mathbf{a}_{I, p} I_{p}(j, n)\right. \\
\left.\quad+\mathbf{H}_{p} \mathbf{E}_{I_{-} \mathrm{UP}, p}(j, n)\right\} \\
=\sum_{p=1}^{P}\left\{\mathbf{a}_{S} S_{p}(j, n)+\mathbf{H}_{p} \mathbf{E}_{I_{-} \mathrm{UP}, p}(j, n)\right\} \\
=\mathbf{E}_{S}(j, n)+\sum_{p=1}^{P} \mathbf{H}_{p} \mathbf{E}_{I_{-} \mathrm{UP}, p}(j, n) .
\end{array}
$$




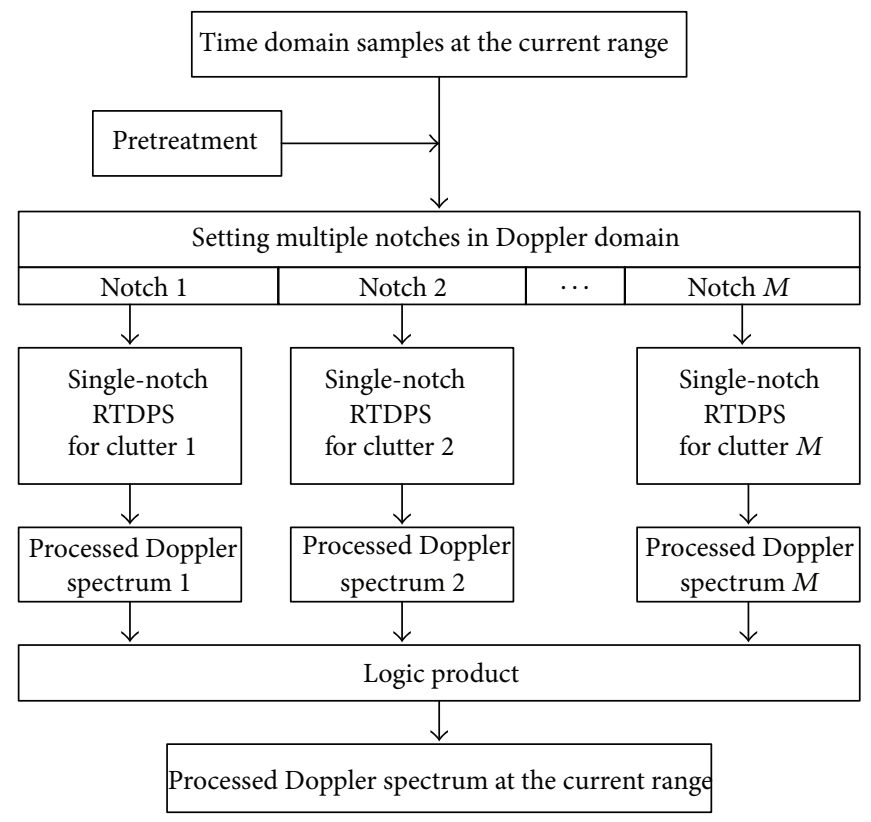

FIgURE 4: Principle of multinotch RTDPS.

After the Doppler processing, the final output of singlenotch RDTPS can be expressed as

$$
\mathbf{Y}_{\mathrm{RTDPS}_{\text {single }}}(j, \omega)=\mathrm{FFT}\left[\mathbf{E}_{S}(j, n)+\sum_{p=1}^{P} \mathbf{H}_{p} \mathbf{E}_{I_{-} \mathrm{UP}, p}(j, n)\right] .
$$

The second component in (35) is the completely unpolarized part of the ionosphere clutter, which will be kept as stochastic noise. Meanwhile, the completely polarized part, described as the first component in (32), can be completely cancelled when $\mathbf{a}_{i, p}$ is accurately estimated.

By dividing the range-time domain data into segments, the estimation and suppression scheme is performed in separate segments and the polarization degree of the ionosphere clutter in each segment can be increased. As a result, the estimation accuracy of the clutter's polarization will be improved and the clutter can be suppressed more completely as the completely unpolarized part is unable to be suppressed.

3.5.2. Multinotch RTDPS. Multinotch RTDPS is implemented for more clutters at the current range cell, as drawn in Figure 4. The quantity and distribution state of the ionosphere clutters at the current range cell are estimated in the pretreatment step. After the locations and sizes of the Doppler notches are decided, single-notch RDTPS is implemented for each clutter. And the final Doppler spectrum is generated by the logic product process.

Supposing the sum of ionosphere clutters is $M, M$ filtering results can be obtained by utilizing single-notch RTDPS shown in (35). Supposing all the clutters are not overlapping with each other in the Doppler spectrum, the result of multinotch RTDPS $\mathbf{Y}_{\text {RTDPS_multi }}(j, \omega)$ can be given as

$$
\begin{aligned}
& \mathbf{Y}_{\text {RTDPS_multi }}(j, \omega) \\
& =\sum_{r=1}^{R}\left\{\delta\left(\omega-\omega_{r}\right) \min _{m=1}^{M}\left(\mathbf{Y}_{\text {RTDPS_single }, m}(j, \omega)\right)\right\},
\end{aligned}
$$

where $M$ and $N$ represent the number of clutters and that of the time domain samples at the current range cell $j$, respectively; $\omega_{r}$ is the frequency of Doppler cell $r$ and $\mathbf{Y}_{\text {RTDPS_single, } m}(j, \omega)$ is the output of the single-notch RTDPS for the $m$ th clutter.

3.5.3. Clutter Polarization Estimation within Each Segment. Supposing the width of the Doppler notch is $K$, the parameters of the samples in this Doppler notch are defined as $\left(\gamma_{I, k, p}, \eta_{I, k, p}, d_{I, k, p}\right), k \in[1, K]$, where the first and second parameters are the polarization angle and angle difference and the third parameter is the power value of the sample. To be clear, the subscript of $I$ denotes the ionosphere clutter, $k$ is the serial number of the Doppler notch, and $p$ represents the serial number of the current segment, respectively.

For each segment, the polarization steering vector of the ionosphere clutter within the Doppler notch can be estimated by

$$
\mathbf{a}_{I, p}\left(\gamma_{I, h, p}, \eta_{I, h, p}\right)=\left[\begin{array}{c}
\cos \gamma_{I, h, p} \\
\sin \gamma_{I, h, p} \exp \left(j \eta_{I, h, p}\right)
\end{array}\right],
$$

where

$$
d_{I, h, p}=\max \left[d_{I, k, p}\right], \quad k, h \in[1, K] .
$$

The estimated values are determined by the sample that has the maximum power within the Doppler notch. 


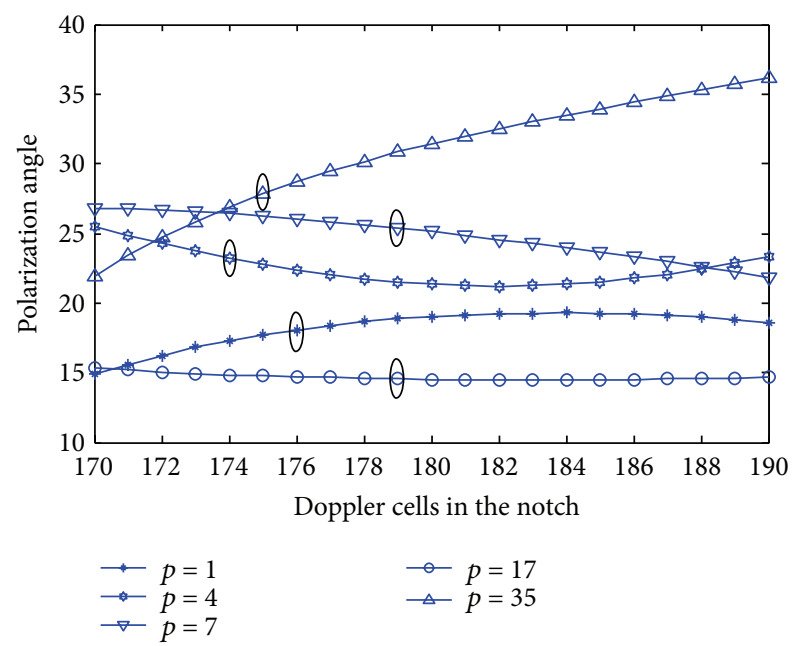

FIGURE 5: Selection of polarization parameters in Doppler notch.

Derived from the experimental HFSWR data, Figure 5 gives polarization angles in segments $1,4,7,17$, and 35 , when total 40 segments are used. The horizontal axis is the Doppler cells whereas the vertical axis is the polarization angle. By using the definition in (38), the samples of the maximum power in the 5 segments are selected and marked with ellipses as shown in Figure 5. According to (37), the polarization steering vectors of each of the corresponding segments can be estimated as $\mathbf{a}_{I, 1}\left(\gamma_{I, 176,1}, \eta_{I, 176,1}\right), \mathbf{a}_{I, 4}\left(\gamma_{I, 174,4}, \eta_{I, 174,4}\right), \mathbf{a}_{I, 7}\left(\gamma_{I, 179,7}, \eta_{I, 179,7}\right)$, $\mathbf{a}_{I, 17}\left(\gamma_{I, 179,17}, \eta_{I, 179,17}\right)$, and $\mathbf{a}_{I, 35}\left(\gamma_{I, 175,35}, \eta_{I, 175,35}\right)$, respectively.

3.5.4. Set of Segment Numbers. In Figure 5, an obvious fluctuating, which is caused by the nonstationary polarization characteristic of the ionosphere clutter, can be found, whereas a plain curve is expected to suppress the clutter with different Doppler frequency together within the notch.

To improve the polarization stationarity or the polarization degree of the clutter in each segment, more segments number should be used. As given in Figure 6, the variance of polarization angle distribution of the clutter decreases when the segment number increases. However, more segments indicate shorter time in coherent integration and result in more estimation error, which degrades the performance of the filter.

Therefore, a satisfied performance of clutter cancellation can be only obtained by properly selecting the number of time domain segment. There is a trade-off between the improvement of polarization degree and reduction of estimation error, which should be balanced in practical application.

\section{Experimental Performance Evaluation}

To evaluate the performance of the proposed method, experimental data of a dual-polarized HFSWR in the east coastal region of China [20] are utilized. The HFSWR is installed by Harbin Institute of Technology and is equipped with

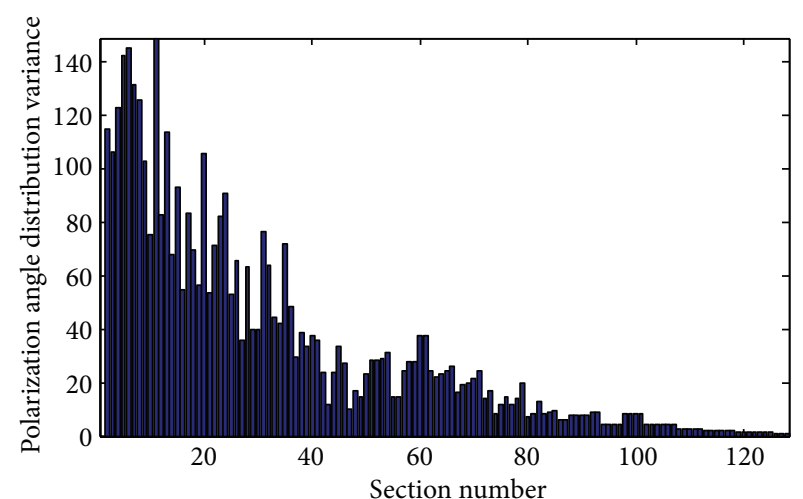

FIGURE 6: Polarization stationarity under different segment numbers.

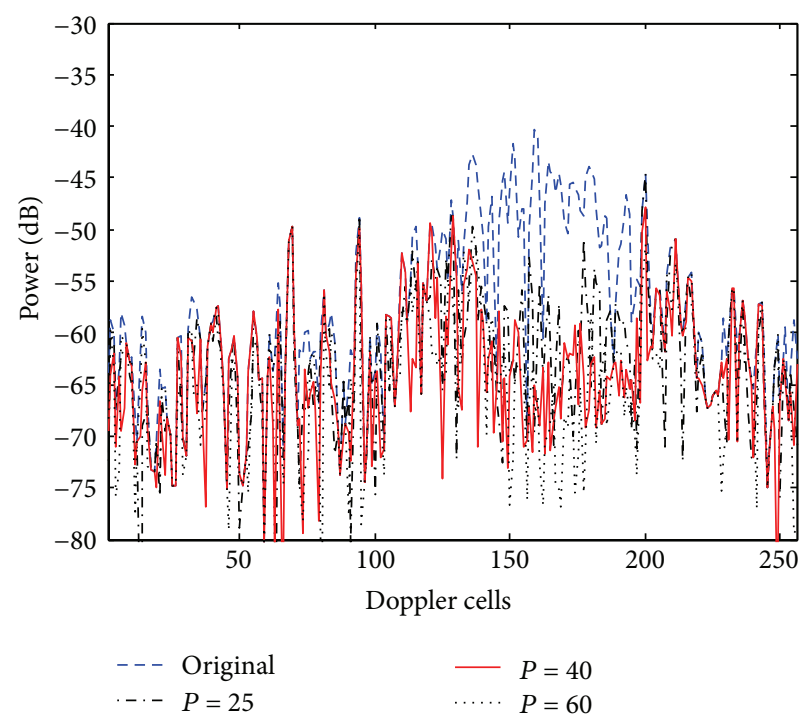

FIGURE 7: Clutter cancellation performance by different segment numbers.

eight-element vertically polarized receiving antenna array and two mutual-orthogonal horizontal polarized antennas to carry out various experiments of interference cancellation.

4.1. Segmentation Setting of RTDPS. Set a group of successive notches that cover the ionosphere clutters region locating in Doppler cells 132-195 at the range cell 157. Figure 7 gives the original Doppler spectrum and the outputs of the RTDPS by using 25,40 , and 60 segments, respectively. The average power of the four curves in the region is $-48 \mathrm{~dB},-61 \mathrm{~dB}$, $-64 \mathrm{~dB}$, and $-66 \mathrm{~dB}$, respectively. It can be found that the clutter can be suppressed effectively, and the suppressed capability depends on the segment number used. The SIR improvement is about $13 \mathrm{~dB}, 16 \mathrm{~dB}$, and $18 \mathrm{~dB}$ when the segment number is 25,40 , and 60 , respectively.

In each segment, the target signal with an ideal polarization will be recovered without any distortion. However, the polarization of some targets may be different with an ideal one and a distortion of amplitude and phase will be introduced 
in these segments. As a result, the targets (or Braggs) are also suppressed when the segment number increases (see Doppler cell 200 in Figure 7).

Therefore, while the segmentation of RTDPS improves the performance of clutter cancellation, the coherent integration of the targets with polarization mismatching will be influenced. Then the segment number should be balanced between the clutter suppressing and target restoration. In our simulation, the segmentation number is set as

$$
P_{\mathrm{opt}}=40 \text {. }
$$

4.2. Ionosphere Clutter Cancellation. Figure 8 gives the result of two typical types of ionosphere clutter suppression at the range cell 69, by the RDDPS and RTDPS, respectively. In Figure 8, the blue dashed line is the original Doppler spectrum, whereas the black solid line and the red dotted line are the results of RDDPS and RTDPS, respectively. In order to evaluate the effectiveness of the clutter cancellation and the target preservation, a synthetic target is also added to the raw data, which locates in the range cell 69 Doppler cell 32 with the power of $-22 \mathrm{~dB}$.

In Figure 8, the peak at the Doppler cell 64 with the power of $-36 \mathrm{~dB}(64,-36 \mathrm{~dB})$ is the left Bragg peak and the one at the Doppler cell 198 with the power of $-30 \mathrm{~dB}(198,-30 \mathrm{~dB})$ is the right Bragg peak of the ocean clutter, whereas the peaks at around $(80,-41 \mathrm{~dB}),(83,-33 \mathrm{~dB}),(118,-45 \mathrm{~dB}),(172,-31 \mathrm{~dB})$, and $(177,-25 \mathrm{~dB})$ are the signals of the targets. At the current range cell, the ionosphere clutter is weak and masks almost all the Doppler cells. In Figure 8, the power of the background noise of RDDPS and RTDPS degrades to about $-73 \mathrm{~dB}$ and $-66 \mathrm{~dB}$, respectively, and the SIR improvement is about $14 \mathrm{~dB}$ for RDDPS and $7 \mathrm{~dB}$ for RTDPS. No matter what methods are used, the targets and Braggs can be well restored and the peaks are clearer after process.

Figure 9 gives the results of RDDPS and RTDPS at the range cell 158, respectively. A synthetic target is also added to the raw data, which locates in the Doppler cell 32 with the power of $-42 \mathrm{~dB}$. Different from the range cell 69 , the ionosphere clutter occupying Doppler cells 110-190 reveals higher power value $(-44 \mathrm{~dB}$ in average), of which the maximum power is as high as $-35 \mathrm{~dB}$, which is $9 \mathrm{~dB}$ higher than the target signal $(94,-44 \mathrm{~dB})$. In Figure 9, the targets at Doppler cells 94 and 32 are completely restored independent of the method. However, the background noise of RDDPS and RTDPS in Doppler cells $110-190$ is about $-59 \mathrm{~dB}$ and $-66 \mathrm{~dB}$, respectively, indicating a better performance of RTDPS.

Figure 10 gives the original range-Doppler map and the final result. The same as in Figures 8 and 9, two synthetic targets at range cells 69 and 158 are added to the raw data. To be specified, a light blue line is attached and divides the original range-Doppler map into two parts. The ionosphere clutter is estimated to be stronger than the targets and Braggs in the upper part, so $\mathrm{Fn}=0$ and RTDPS is applied in the range cells 156-200, whereas Fn $=1$ in the lower part of the map and the RDDPS is utilized in the rest of range cells. Processed with the proposed method, the weak ionosphere clutter that masks range cells $25-155$ is suppressed close to the power of background noise, and the SIR improvement is about $15 \mathrm{~dB}$

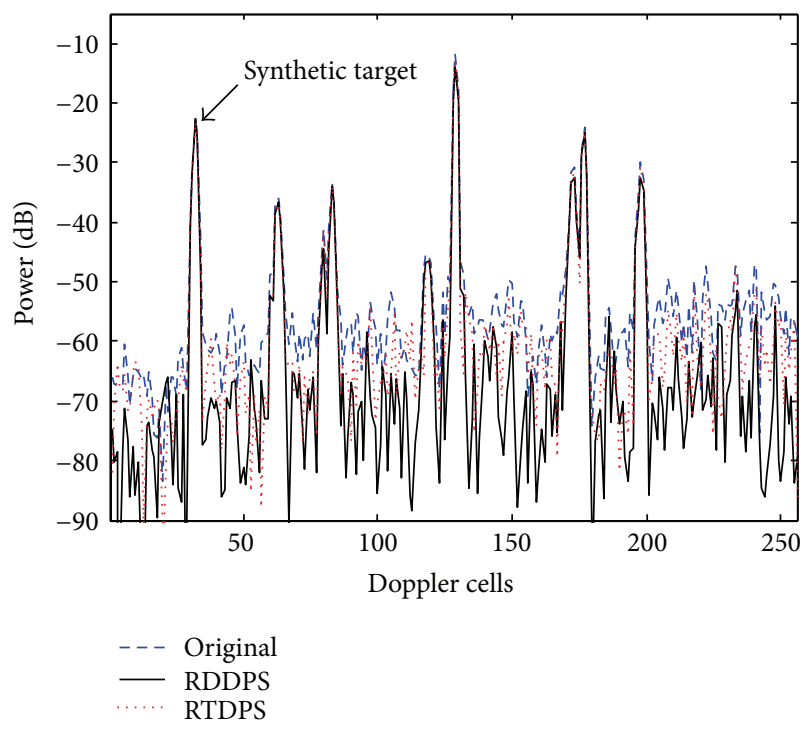

Figure 8: Results of RDDPS and RTDPS at range cell 69.

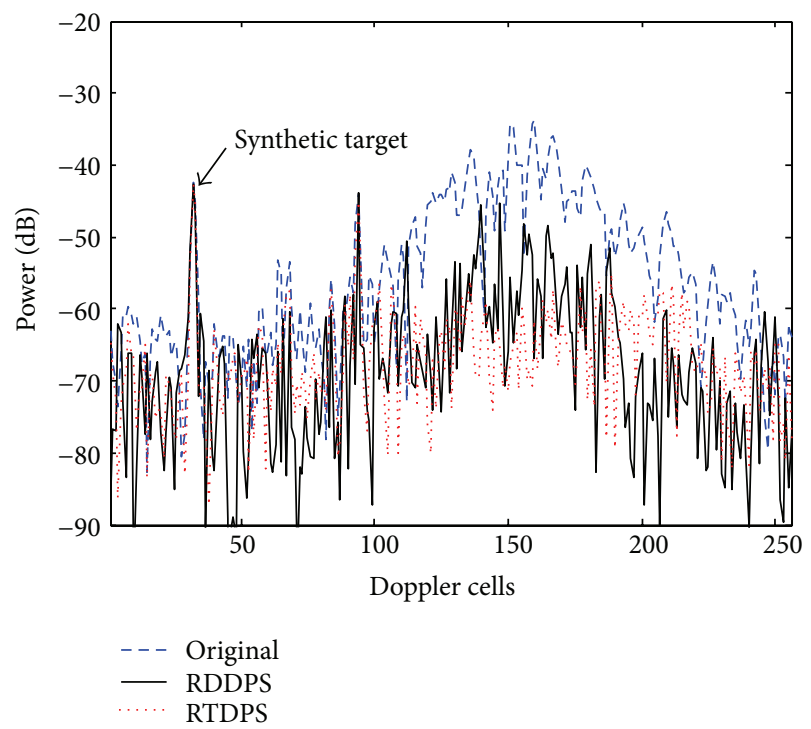

Figure 9: Results of RTDPS at range cell 158.

in average. Meanwhile, the powerful ionosphere clutters that cover range cells from 156 to 180 are also suppressed, while the SIR improvement is about $22 \mathrm{~dB}$ in average. As a result, all the targets are more clear and easier to be detected and tracked in the range-Doppler map.

\section{Conclusion}

In this paper, a novel ionosphere clutter cancellation scheme is proposed. Two types of ionosphere clutter cancellation methods, RDDPS and RTDPS, are designed based on the theory of OPPF. RTDPS is suitable in dealing with powerful ionosphere clutter, whereas the RDDPS performs a stable filtering effect to suppress the clutter of low power. The selection of RDDPS or RTDPS algorithm is determined by 


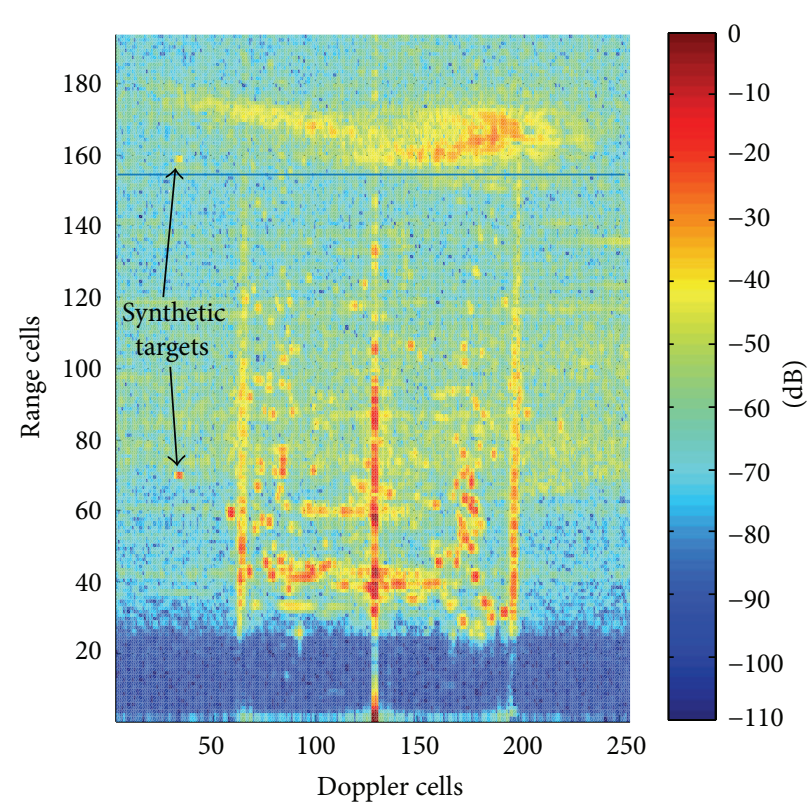

(a)

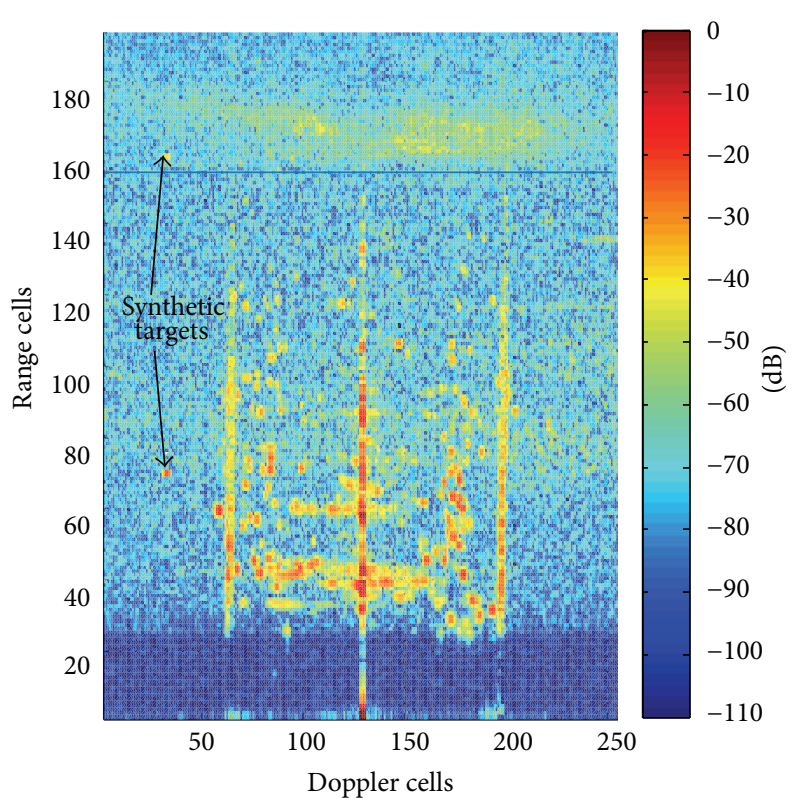

(b)

FIGURE 10: The original and the processed range-Doppler map.

the clutter type at the corresponding range cell. Combining the RDDPS and RTDPS together, the ionosphere clutters can be suppressed deeply. Theoretical analysis and experimental results demonstrate that the proposed scheme is valid and the SIR can be increased more than $15 \mathrm{~dB}$. It is indicated that the proposed method may serve as a suitable method for ionosphere clutter cancellation in a practical HFSWR system.

\section{Conflict of Interests}

The authors declare that there is no conflict of interests regarding the publication of this paper.

\section{Acknowledgment}

This project is sponsored by the National Natural Science Foundation of China (no. 61171180).

\section{References}

[1] H. C. Chan, "Characterization of ionospheric clutter in HF surface wave radar," Defence R\&D Canada-Ottawa Technical Report, DRDC Ottawa TR, 2003.

[2] W. Shen, B.-Y. Wen, Z.-L. Li, X.-J. Huang, and J. Yang, "Ionospheric measurement with HF ground wave radar system," Chinese Journal of Radio Science, vol. 23, no. 1, pp. 1-5, 2008.

[3] X. Guo, H. Sun, and T. S. Yeo, "Interference cancellation for high-frequency surface wave radar," IEEE Transactions on Geoscience and Remote Sensing, vol. 46, no. 7, pp. 1879-1891, 2008.

[4] H.-T. Su and Z. Bao, "Adaptive HF-communication interference mitigation in HF-GWR," Chinese Journal of Radio Science, vol. 18, no. 3, pp. 270-274, 2003.
[5] G. A. Fabrizio, A. B. Gershman, and M. D. Turley, "Robust adaptive beamforming for HF surface wave over-the-horizon radar," IEEE Transactions on Aerospace and Electronic Systems, vol. 40, no. 2, pp. 510-525, 2004.

[6] A. J. Poelman, "Virtual polarisation adaptation-a method of increasing the detection capability of a radar system through polarization-vector processing," IEE Proceedings of Communications, Radar and Signal Processing, vol. 128, no. 5, pp. 261-270, 1981.

[7] M. Gherardelli, D. Giuli, and M. Fossi, "Suboptimum adaptive polarisation cancellers for dual-polarisation radars," IEE Proceedings, Part F: Communications, Radar and Signal Processing, vol. 135, no. 1, pp. 60-72, 1988.

[8] G. Y. Zhang and Y. T. Liu, "Study of the polarization filtering technique of HF ground wave radar," Journal of Systems of Engineering and Electronics, vol. 22, no. 3, pp. 55-57, 2000.

[9] R. Tian and X. Tian, "Novel polarization filter design for wideband radar," Journal of Systems Engineering and Electronics, vol. 23, no. 4, pp. 522-528, 2012.

[10] X.-P. Mao, Y.-T. Liu, W.-B. Deng, C.-J. Yu, and Z.-L. Ma, "Null phase-shift instantaneous polarization filter," Acta Electronica Sinica, vol. 32, no. 9, pp. 1495-1498, 2004.

[11] X.-P. Mao and Y.-T. Liu, "Null phase-shift polarization filtering for high-frequency radar," IEEE Transactions on Aerospace and Electronic Systems, vol. 43, no. 4, pp. 1397-1408, 2007.

[12] X.-P. Mao, Y.-T. Liu, and W.-B. Deng, "Frequency domain null phase-shift multinotch polarization filter," Acta Electronica Sinica, vol. 36, no. 3, pp. 537-542, 2008.

[13] X.-P. Mao, A.-J. Liu, W.-B. Deng, B. Cao, and Q.-Y. Zhang, "An oblique projecting polarization filter," Acta Electronica Sinica, vol. 38, no. 9, pp. 2003-2008, 2010.

[14] X.-P. Mao, A.-J. Liu, and H.-J. Hou, "Oblique projection polarisation filtering for interference suppression in high-frequency surface wave radar," IET Radar, Sonar and Navigation, vol. 6, no. 2, pp. 71-80, 2012. 
[15] R. T. Behrens and L. L. Scharf, "Signal processing applications of oblique projection operators," IEEE Transactions on Signal Processing, vol. 42, no. 6, pp. 1413-1424, 1994.

[16] K. Davies, Ionospheric Radio, pp. 225-230, Peter Peregrinus, London, UK, 1990.

[17] M. Xingpeng, L. Yongtan, D. Weibo, Y. Changjun, and M. Zilong, "Sky wave interference of high-frequency surface wave radar," Electronics Letters, vol. 40, no. 15, pp. 968-969, 2004.

[18] S. Leinwoll, Shortwave Propagation, John Lyder Publisher, New York, NY, USA, 1959.

[19] A. Roueff, J. Chanussot, and J. I. Mars, "Estimation of polarization parameters using time-frequency representations and its application to waves separation," Signal Processing, vol. 86, no. 12, pp. 3714-3731, 2006.

[20] Y. T. Liu, R. Q. Xu, and N. Zhang, "Progress in HFSWR research at Harbin institute of technology," in Proceeding of the IEEE Radar Conference, pp. 522-528, Adelaide, Australia, September 2003. 

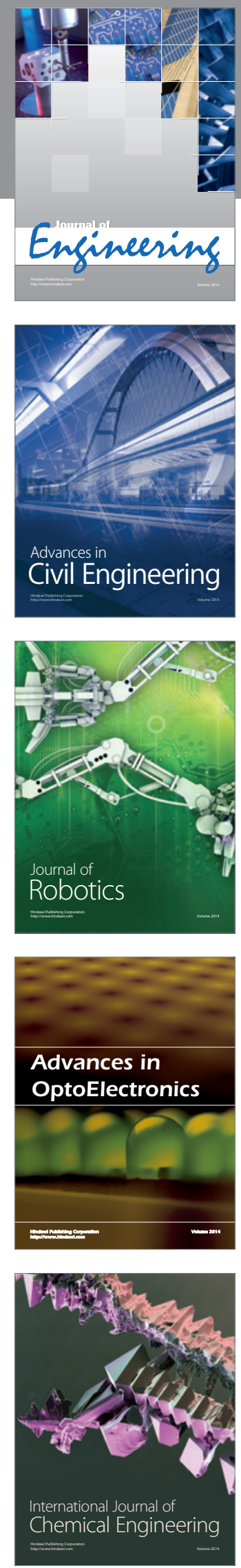

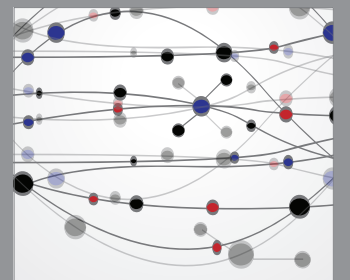

The Scientific World Journal
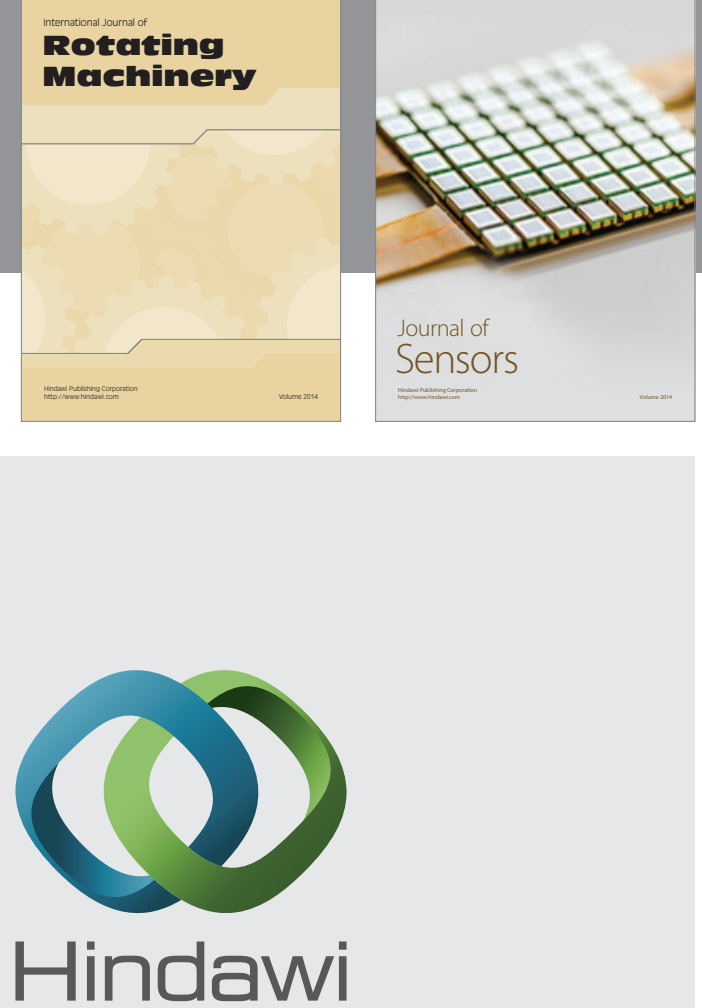

Submit your manuscripts at http://www.hindawi.com
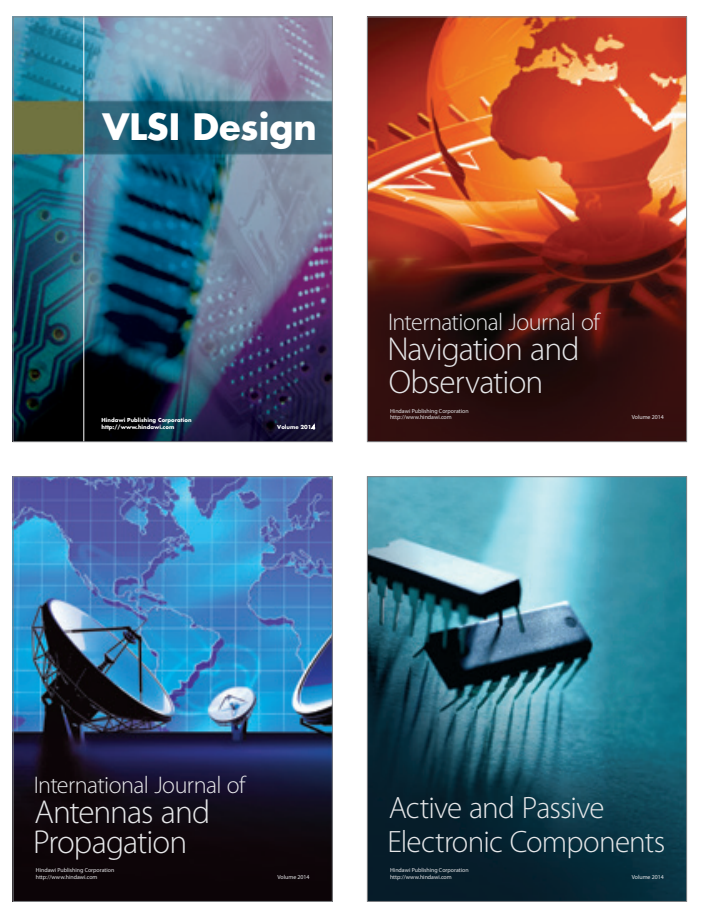
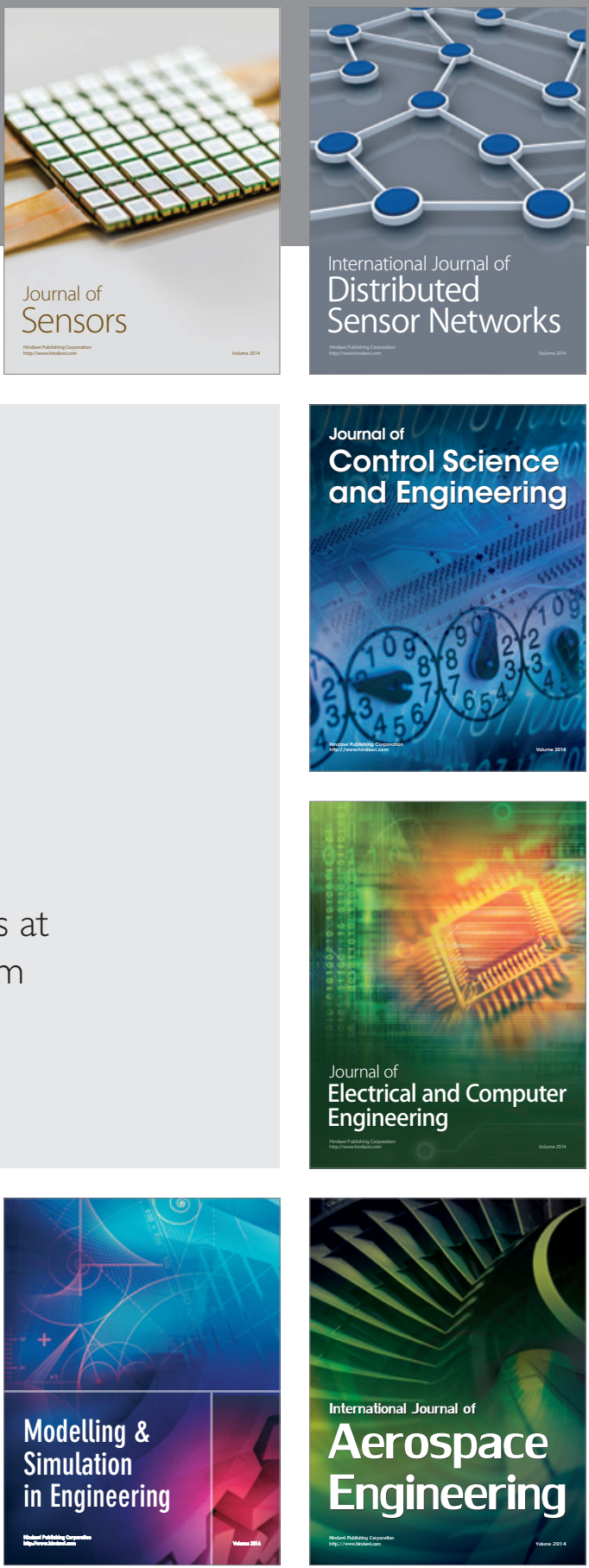

Journal of

Control Science

and Engineering
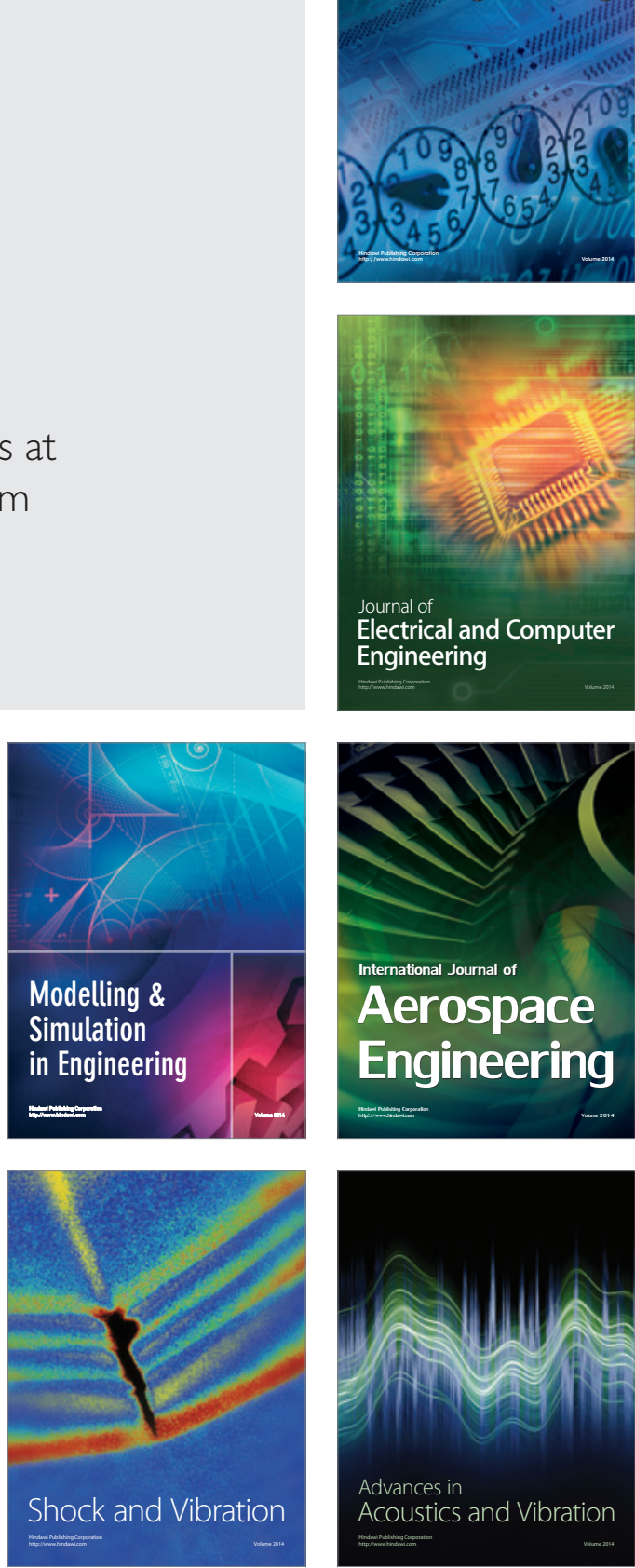